\title{
3D TrueFISP MRI Provides Accurate Longitudinal Measurements of Glioma Volumes in Mice
}

\author{
Emeline Julie Ribot ${ }^{1,2}$, Line Pourtau ${ }^{1,3}$, Philippe Massot ${ }^{1,3}$, Pierre Voisin ${ }^{1,3}$, \\ Eric Thiaudiere ${ }^{1,3}$, Jean- Michel Franconi ${ }^{1,3}$ and Sylvain Miraux ${ }^{1,3}$ \\ ${ }^{1}$ Centre de Résonance Magnétique des Systèmes Biologiques, \\ Université Bordeaux Segalen, CNRS \\ ${ }^{2}$ Imaging Research Laboratories, Robarts Research Institute, \\ The University of Western Ontario \\ ${ }^{3}$ Laboratory of Excellence TRAIL, Translational Research and Advanced Imaging \\ Laboratory, University of Bordeaux \\ 1,3 France \\ ${ }^{2}$ Canada
}

\section{Introduction}

One of the most common models used in pre-clinical studies is nude mice implanted with brain tumors. In fact, owing to a deficiency in their immune systems, nude mice allow many tumor models to be studied and a wide range of therapeutic treatments to be investigated.

The best way to evaluate the efficiency of a treatment is to compare the kinetics of the tumor volume between a control group and a treatment group [1-3]. Since brain tumors generally evolve very quickly ( 7 to 15 days) in mice, the tumor must be detected early and characterized daily.

The advantages of non-invasive imaging methods are evident compared with conventional methods such as histochemistry, where animal sacrifice, end-point analysis only, and 2D tumor-diameter measurement are mandatory.

However, obtaining images of high spatial resolution and high contrast for the same animal in a longitudinal study is not trivial.

As in clinical MR imaging, the tumor can be detected in two possible ways. The first consists of using a gadolinium-based contrast agent injected intravenously to visualize the breakdown of the blood-brain barrier. Using a T1-weighted 3D gradient-echo sequence, high-resolution images can be obtained in a reasonable acquisition time. Nevertheless, because of the injuries caused by repeated injection of contrast agent into the tail vein, experiments cannot be performed frequently [1]. The other way consists of using T2weighted sequences. RARE imaging, with or without magnetization transfer preparation [35], allows small tumors to be detected noninvasively. The sequence is usually acquired in multi-slice 2D imaging in order to limit the total acquisition time. However, high-resolution, 
accurate 3D information is very important in longitudinal studies such as, for instance, the evaluation of a therapeutic treatment.

An alternative to RARE T2 [6] or 3D contrast-enhanced T1 imaging is fully balanced SSFP imaging (also called bSSFP, TrueFISP or FIESTA) [7]. In fact, it has recently been shown that this sequence can be used at high field, in 3D, to detect tumors of very small size [8]. The advantage of this sequence is the ability to combine the speed of $3 \mathrm{D}$ gradient echo and T1/T2 contrast.

The purpose of this study is to demonstrate that a 3D TrueFISP MRI sequence is applicable, at high magnetic field, to glioma-bearing mouse models in longitudinal studies. Theoretical considerations of tumor contrast and signal-to-noise ratio as a function of sequence parameters (TE/TR/flip angle) were carried out and compared with experimental data.

The 3D TrueFISP MRI sequence was also compared with the sequence most widely used in clinical applications: 2D RARE. Finally, the sequence was used to perform accurate longitudinal measurements of glioma volumes in mice.

\section{Materials and methods}

\subsection{MRI system}

\section{Magnet}

NMR machines to image small animals are becoming more and more widespread. The various manufacturers have developed systems for use in studies using small animals by adapting their components (magnet, gradients, antennae, monitoring system and animal handling solutions).

The main component of an MRI system is the magnet, which generates an intense static magnetic field. While for clinical imaging 1.5-T and 3-T magnets are used, for rats and mice a magnetic field between $4.7 \mathrm{~T}$ and $11.6 \mathrm{~T}$ is generally used. The magnets can be positioned either horizontally or vertically. This type of magnetic fields provides an increased signal-to-noise ratio $(\mathrm{S} / \mathrm{N})$, required for the acquisition of highly spatially resolved images. However, a theoretical increase in signal causes problems which must be considered when high-quality images are to be obtained. In this way, an increase in magnetic field leads to a reduced natural contrast between the tissues in T2 or T1weighted scans. In addition, susceptibility effects are enhanced and may lead to artifacts with gradient-echo sequences.

Most of the images presented here were acquired at $4.7 \mathrm{~T}$. This level of magnetic field provides a compromise between signal-to-noise ratio, contrast and sensitivity to susceptibility artifacts. The use of a more intense magnetic field will also be addressed.

\subsection{Gradient system}

The second essential component of an MRI system is the magnetic field gradient. Gradients allow the image to be spatially encoded. When imaging small animals, the spatial resolution required is much greater than for human imaging. For example, in anatomic imaging of rats, a voxel size of at least $400 \mu \mathrm{m}$ is required in all three dimensions (3D); in mice, a size of at least $200 \mu \mathrm{m}$ is needed.

In MRI, the digital resolution, i.e. pixel size, is inversely proportional to the gradient intensity, G, and application time, tG (Eq. [1]). 


$$
\Delta y=\frac{\pi}{t_{G} \gamma G}
$$

The actual resolution is inversely proportional to the relaxation time, $\mathrm{T} 2 *$, and to the gradient intensity and application time (Eq. [2]).

$$
\text { Resolution }=\frac{2}{\gamma \mathrm{GT}_{2}{ }^{*}}
$$

The advantages of using intense magnetic field gradients are thus obvious, particularly at strong magnetic fields, with very short $\mathrm{T} 2 *$.

Commercial gradient systems have been developed with intensities of greater than $400 \mathrm{mT} / \mathrm{m}$ over $10 \mathrm{~cm}$, up to $1 \mathrm{~T} / \mathrm{m}$ over $3 \mathrm{~cm}$. Because the target diameter of these gradient systems is spatially limited, they can only be used to study animals such as rats or mice.

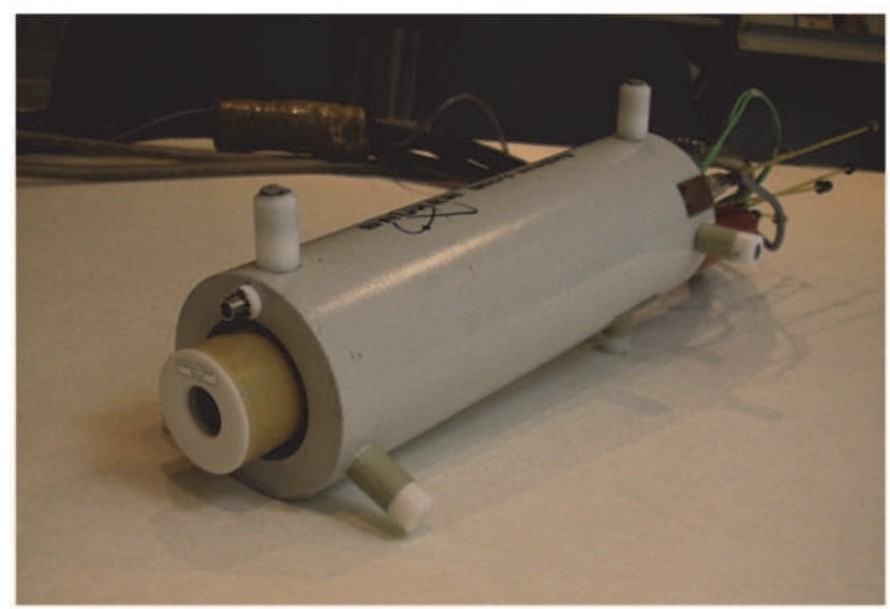

Fig. 1. Photograph of a 1-T/m gradient system. The system can be inserted into a magnet equipped with a 12-cm-diameter tunnel and can contain an antenna up to $6 \mathrm{~cm}$ in diameter. The gradient is linear over a distance of approximately $3 \mathrm{~cm}$.

\subsection{Coil}

Image quality is also significantly affected by the choice of antenna. The S/N depends entirely on this component as it is proportional to the square root of the antenna's quality factor, Q. Thus, a well-designed antenna is recommended. In addition, the $\mathrm{S} / \mathrm{N}$ is directly proportional to the filling factor for the antenna, represented by $\eta$ in Eq. [3].

$$
\mathrm{S} / \mathrm{N} \propto(\mathrm{Q})^{1 / 2} \eta
$$

Optimal image quality will therefore be obtained using an antenna with a size and shape perfectly adapted to the zone to be imaged. Several types of antennae have been developed and can be used to meet these criteria. Volumetric emission/reception antennae are the most common. They provide a very homogeneous radio-frequency field, often required when 
using spin-echo sequences. However, their filling factor is relatively low, which can penalize the $\mathrm{S} / \mathrm{N}$. This sensitivity problem can be partially mitigated by using cross-polarized antennae. This type of volumetric antenna is the easiest to use, and was used for the images presented in this chapter.

Surface antennae can also be used, offering an excellent quality factor combined with a very high filling factor. This type of antenna therefore provides a higher $\mathrm{S} / \mathrm{N}$ than the antennae described previously.

However, due to their configuration, the flip angle applied varies depending on the depth of the zone to be imaged within the sample, making the use of spin-echo sequences impossible. In addition, with this type of antenna, contrast can vary depending on the depth of the zone observed within the sample.

Finally, in clinical imaging, and increasingly for small-animal imaging, volumetric emission antennae coupled to an array of surface reception antennae are the most commonly used. This setup offers the advantages of the two types of antennae described above: excellent B1 emission homogeneity associated with an excellent signal-to-noise ratio.
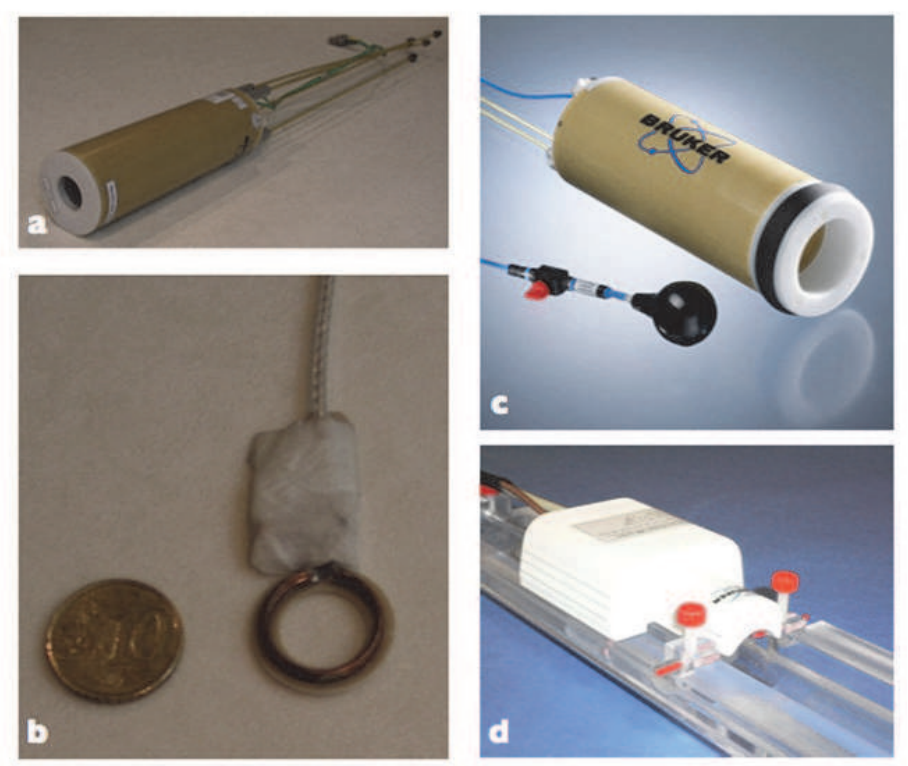

Fig. 2. a) Cross-polarized 25-mm-diameter volumetric antenna; b) 16-mm-diameter surface emission/reception antenna; c) \& d) antenna system combining volumetric emission ( $80 \mathrm{~mm}$ in diameter) and phased-array reception.

These three types of antennae are exclusively used for mouse brain imaging and were tuned to $200 \mathrm{MHz}$ in the experiments described here.

\subsection{Animal handling/monitoring}

Finally, quality imaging in small animals requires a specific component to position and monitor the animal. This component must allow the animal to be maintained in a stable and 
fairly reproducible position. In particular, it must allow imaging to be applied repeatedly (approximately once a day) to particularly fragile animals.

A tooth bar assembly is generally used to position the animal's head based on the position of its teeth. Ear bars may be added but are not always necessary. This type of stereotactic positioning is often used for brain imaging in animals.

The animal is monitored by measuring its respiratory rate using an air balloon placed on the abdomen or back of the animal and connected to a pressure sensor linked to a computer via an optical fiber during imaging sessions. The respiratory rate can thus be followed on a computer screen in real time.

Isoflurane inhalation-based anesthesia is used, with adaptation of the percentage of isoflurane mixed with air to maintain the animal's respiration rate at greater than 70 and less than 110 breaths/min. Isoflurane can be recycled through a capture system.

The animal's body temperature is maintained constant either with an electric blanket, or by using circulating hot water to maintain the gradient system at $32{ }^{\circ} \mathrm{C}$.
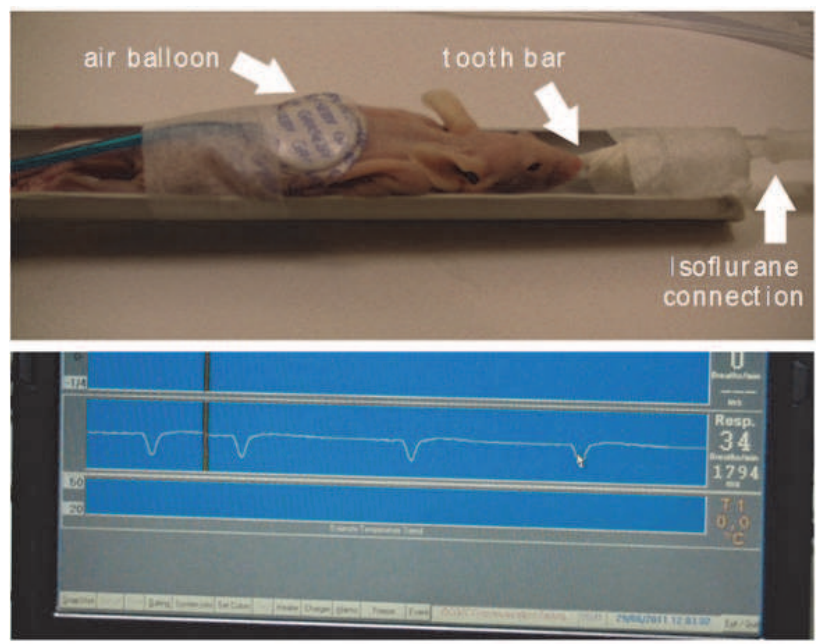

Fig. 3. Nude mouse in a home-made bed. A tooth-bar assembly holds the animal in position and a 1-2\% air/isoflurane mixture is delivered in the region of the nose. A balloon is placed on the animal's back to measure its respiration rate. The SA Instruments system is linked to a computer, where the animal's respiration can be visualized on-screen.

\subsection{Animal model}

U87 human brain tumor cells were implanted in nude mice (18-20 g, $n=20$, Charles Rivers, L'Arbresle, France) by stereotactic injection into the striatum. Mice were anesthetized with isoflurane (1.5\% in air) and secured in the stereotactic apparatus (Stoelting Europe, Dublin, Ireland). The scalp was cleaned with Betadine (MEDA Pharma, Paris, France) and the skull was exposed by midline scalp excision. A small hole $(0.5 \mathrm{~mm}$ in diameter $)$ was then drilled $0.1 \mathrm{~mm}$ posterior and $2.3 \mathrm{~mm}$ left to the bregma. Five hundred thousand U87 cells dissolved in $2 \mathrm{~mL}$ of Minimum Essential Medium were injected using a 10-mL Hamilton syringe into the left hemisphere at a depth of $3 \mathrm{~mm}$ below the brain's surface. On withdrawal of the injection needle, the hole in the skull was sealed with bone wax and the scalp was sutured. 


\section{Sequence}

\subsection{RARE 2D}

T2-weighted sequences are the most widely used for imaging-based tumor detection. These are accelerated spin-echo sequences (RARE, or Rapid Acquisition with Refocused Echoes) which are generally acquired in two dimensions (2D), in multi-slice mode. When these sequences are used for clinical imaging, pulses are often added to suppress fluid signals (FLAIR, or Fluid-Attenuated Inversion Recovery).

To image small animals, the following parameters are used:

in $2 \mathrm{D}$

TE/TR $=70 / 5,000 \mathrm{~ms} ; \quad$ FOV: $22.5 \times 22.5 \mathrm{~mm}$; matrix: $192 \times 128 ;$ spatial resolution: $117 \times 175 \mu \mathrm{m}$; slice thickness: $750 \mu \mathrm{m}$; excitation pulse: Hermite $1 \mathrm{~ms}, 90^{\circ}$; refocusing pulse: Hermite $1 \mathrm{~ms}, 180^{\circ}$; reception bandwidth: $260 \mathrm{Hertz} /$ pixel; RARE factor: 32; number of averages: 32 , total acquisition time: $10 \mathrm{~min} 40 \mathrm{sec}$; transverse orientation.

\subsection{TrueFISP}

The specificity of bSSFP (balanced Steady-State Free Precession) type sequences, also known as TrueFISP or FIESTA, is their perfect symmetry, with an echo time (TE) equal to half the repetition time (TR). This maintains the magnetization in the transverse plane. Due to spin dephasing, the signal generated is more intense than with gradient-echo sequences. This allows 3D images to be constructed with good spatial resolution within a reasonable acquisition time. Besides, the tissue contrast generated is proportional to the T1/T2 ratio. The alternating phase RF pulse method must be used with 4 or $8 \Delta \varphi$ phase values $\left(180^{\circ}, 0^{\circ}\right.$, $90^{\circ}, 270^{\circ} ; 45^{\circ}, 135^{\circ}, 225^{\circ}, 315^{\circ}$ ). Image reconstruction is based on the Sum-Of-Square (SOS) method, i.e. calculation of the square root of the sum of squares of the magnitude signal.

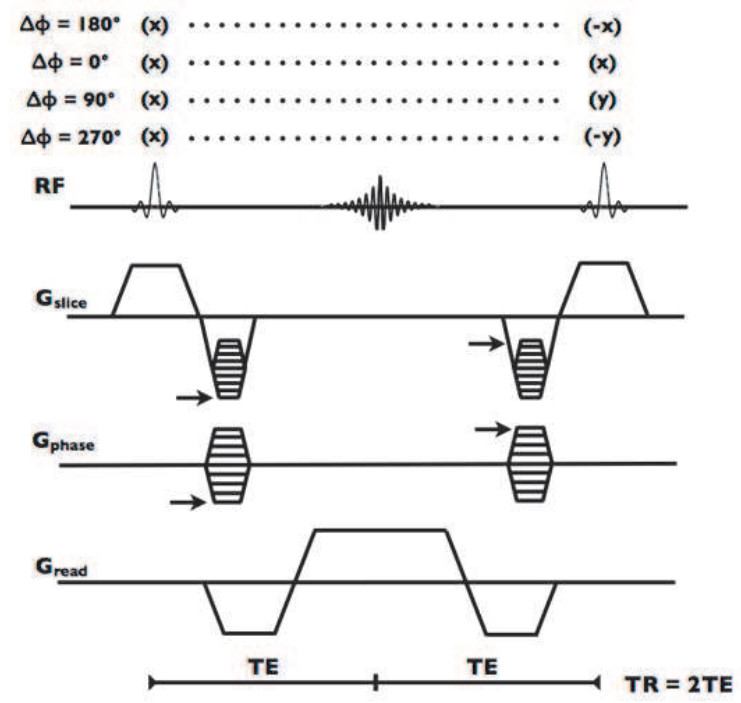

Fig. 4. 3D TrueFISP sequence chronogram. The RF pulse phase indicated generates images corresponding to the following $\Delta \varphi$ values: $180^{\circ}, 0^{\circ}, 90^{\circ}, 270^{\circ}$. The sequence is perfectly symmetrical about the center of the echo. Thus, the echo time (TE) is equal to half the repetition time (TR). 
The following sequence parameters are used:

- $\quad$ TE/TR = 2.5/5 ms; FOV: $32 \times 18 \times 18$ mm; matrix: $192 \times 96 \times 80$; spatial resolution: $166 \times 187 \times 225 \mu \mathrm{m}$; excitation pulse: Hermite $0.4 \mathrm{~ms}$; reception bandwidth: $260 \mathrm{Hertz} /$ pixel; number of averages (NA): 16 (including $4 \Delta \varphi$ values), total acquisition time: $2 \mathrm{~min} 34 \mathrm{sec} \times 4=10 \mathrm{~min} 16 \mathrm{sec}$; transverse orientation.

- $\quad \mathrm{TE} / \mathrm{TR}=1.5 / 3 \mathrm{~ms}$;

- $\quad \mathrm{TE} / \mathrm{TR}=5 / 10 \mathrm{~ms} ;$

- $\quad$ TE/TR = 10/20 ms;

\subsection{Theory}

Simulations of the state of magnetization with TrueFISP, gradient-echo and spin-echo sequences were performed in Igor Pro using well-known equations (Eq. [4], [5] and [6)]. They were performed as a function of flip angle $a, \mathrm{TR}, \mathrm{TE}$ or the T1 and T2 values of tissues. At $4.7 \mathrm{~T}$, the $\mathrm{T} 1$ and $\mathrm{T} 2$ values of brain was equal to $1,295 \mathrm{~ms}$ and $53 \mathrm{~ms}$, respectively, and for tumors, 1,525 and $72 \mathrm{~ms}$, respectively [8].

- TrueFISP

$$
\mathrm{S}_{\text {TrueFISP }}=\frac{\left(1-\exp ^{-\mathrm{TR} / \mathrm{T} 1}\right) \sin \alpha}{1-\left(\exp ^{-\mathrm{TR} / \mathrm{T} 1}-\exp ^{-\mathrm{TR} / \mathrm{T} 2}\right) \cos \alpha-\left(\exp ^{-\mathrm{TR} / \mathrm{T} 1} \exp ^{-\mathrm{TR} / \mathrm{T} 2}\right)} \exp ^{-0.5 \mathrm{TR} / \mathrm{T} 2}
$$

- Gradient echo

$$
\mathrm{S}_{\mathrm{GE}}=\frac{\left(1-\exp ^{-\mathrm{TR} / \mathrm{T} 1}\right) \sin \alpha}{1-\cos \alpha\left(\exp ^{-\mathrm{TR} / \mathrm{T} 1}\right)} \exp ^{-\mathrm{TE} / \mathrm{T} 2^{*}}
$$

with $\mathrm{TE}=2.5 \mathrm{~ms}$ and $\mathrm{T} 2{ }^{*}=25 \mathrm{~ms}$

- Spin echo

A 1/6.32 correction factor was applied to 2D RARE (Eq. [6]), corresponding to the voxel size, the number of averages and the number of k-space samples by comparison with 3D TrueFISP acquisition.

$$
\left.\mathrm{S}_{\mathrm{SE}}=\left(1-2 \exp ^{(\mathrm{TE} / 2-\mathrm{TR}) / \mathrm{T} 1}\right)+\exp ^{-\mathrm{TR} / \mathrm{T} 1}\right) \exp ^{-\mathrm{TE} / \mathrm{T} 2}
$$

$\mathrm{S} / \mathrm{N}$ was simulated for the brain and tumor, and contrast was evaluated according to Eq. [7].

$$
\text { Contrast }=\mathrm{S}_{\text {Tumor }}-\mathrm{S}_{\text {Brain }}
$$

The signal-to-noise ratio was evaluated for both tumor and brain tissues. For the TrueFISP sequence, the maximal signal was obtained with the shortest possible TE and a flip angle between approximately $15^{\circ}$ and $35^{\circ}$, as shown in Figs. 5ab. The slight S/N difference between the healthy brain and the tumor is also observable. The gradient-echo sequence generates a much lower $\mathrm{S} / \mathrm{N}$ compared with the TrueFISP sequence for all flip angles. 

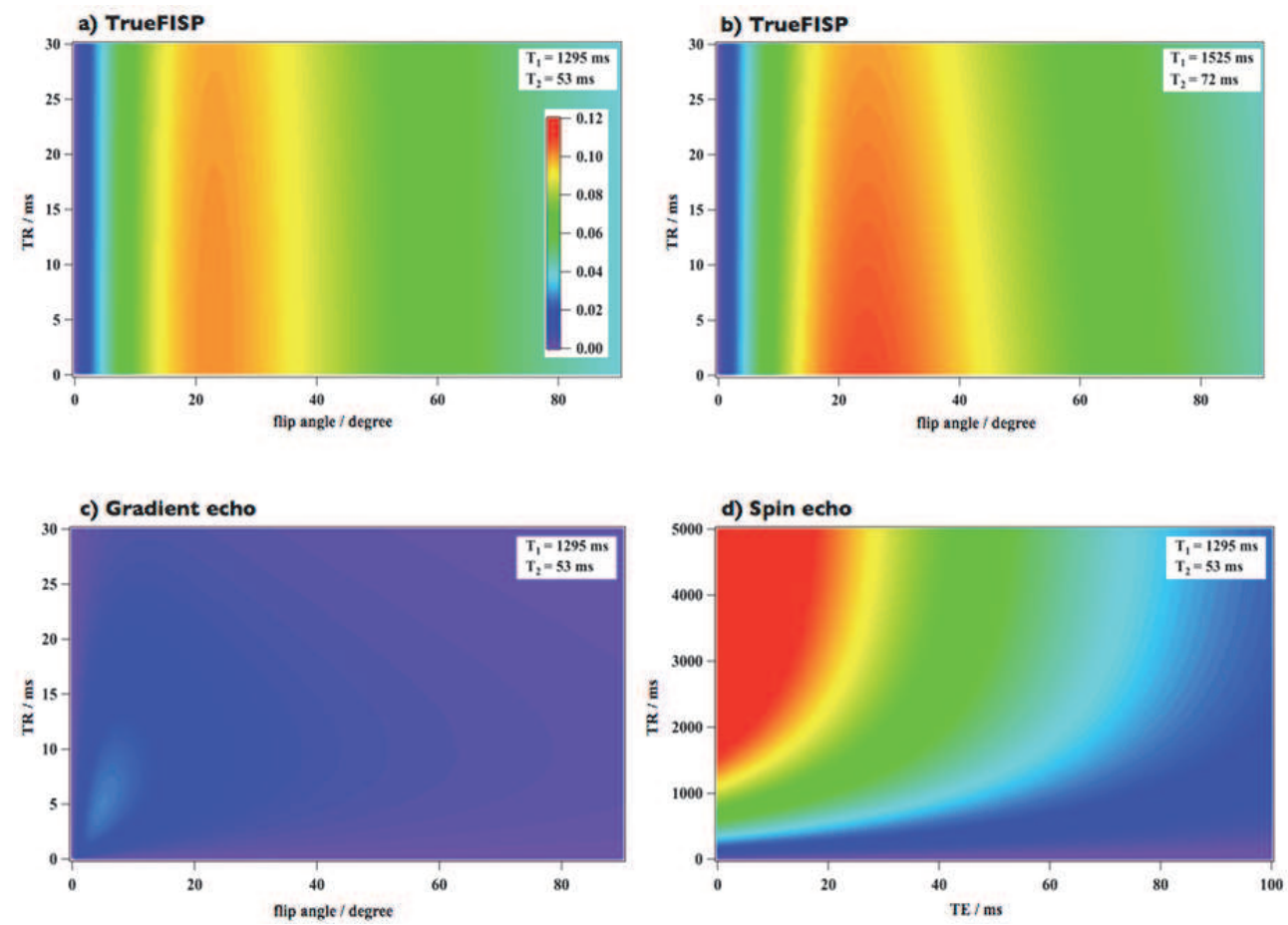

Fig. 5. Modeling of the signal-to-noise ratio using the above equations for: ab) the TrueFISP sequence, c) the gradient-echo sequence, and d) the spin-echo sequence. The signal was estimated as a function of the flip angle and TR for a), b), and c). For the spin-echo sequence, the signal was estimated as a function of the TEs and TRs of the sequence. The T1 and T2 values for the brain (1,295 ms and $53 \mathrm{~ms}$, respectively) were used in a), c), and d). The T1 and $\mathrm{T} 2$ values for the tumor $(1,525 \mathrm{~ms}$ and $72 \mathrm{~ms}$, respectively) were used in $\mathrm{b})$. The same color scale was used in the 4 simulations. To allow for the 2-dimensional nature of the spinecho sequence, a correction factor was applied.

The spin-echo sequence should provide a high $\mathrm{S} / \mathrm{N}$, provided the TR is long and TE very short. However, these acquisition conditions do not provide sufficient contrast, as shown in the following figure.

We can thus clearly see the advantage of the TrueFISP sequence, as far as the $\mathrm{S} / \mathrm{N}$ is concerned, for an equivalent total acquisition time, compared with both a standard 3D gradient-echo sequence and a 2D RARE spin-echo sequence.

\subsection{Contrast}

The contrast was then assessed theoretically, based on the signal difference between the tumor and the healthy brain. For the TrueFISP sequence (Fig. 6a), optimal contrast was achieved with a short TE and a flip angle between $25^{\circ}$ and $50^{\circ}$.

The gradient-echo sequence provided almost no contrast compared with the TrueFISP sequence, while the spin-echo sequence provided improved contrast for TRs over 3,500 ms and TEs greater than $50 \mathrm{~ms}$. 
To conclude on these simulations (S/N and contrast), a very high $\mathrm{S} / \mathrm{N}$ can be expected with the TrueFISP sequence. This method provides adequate contrast between the brain and tumor with flip angles around $30^{\circ}$ and short TEs. Yet, contrast should be improved with the T2-weighted accelerated spin-echo sequence (RARE), but this method is generally used to acquire 2D images only, thus limiting its capacity to measure tumor volumes. Using it for 3D imaging can produce artifacts due to movements, and the acquisition time is much longer than with the TrueFISP sequence.
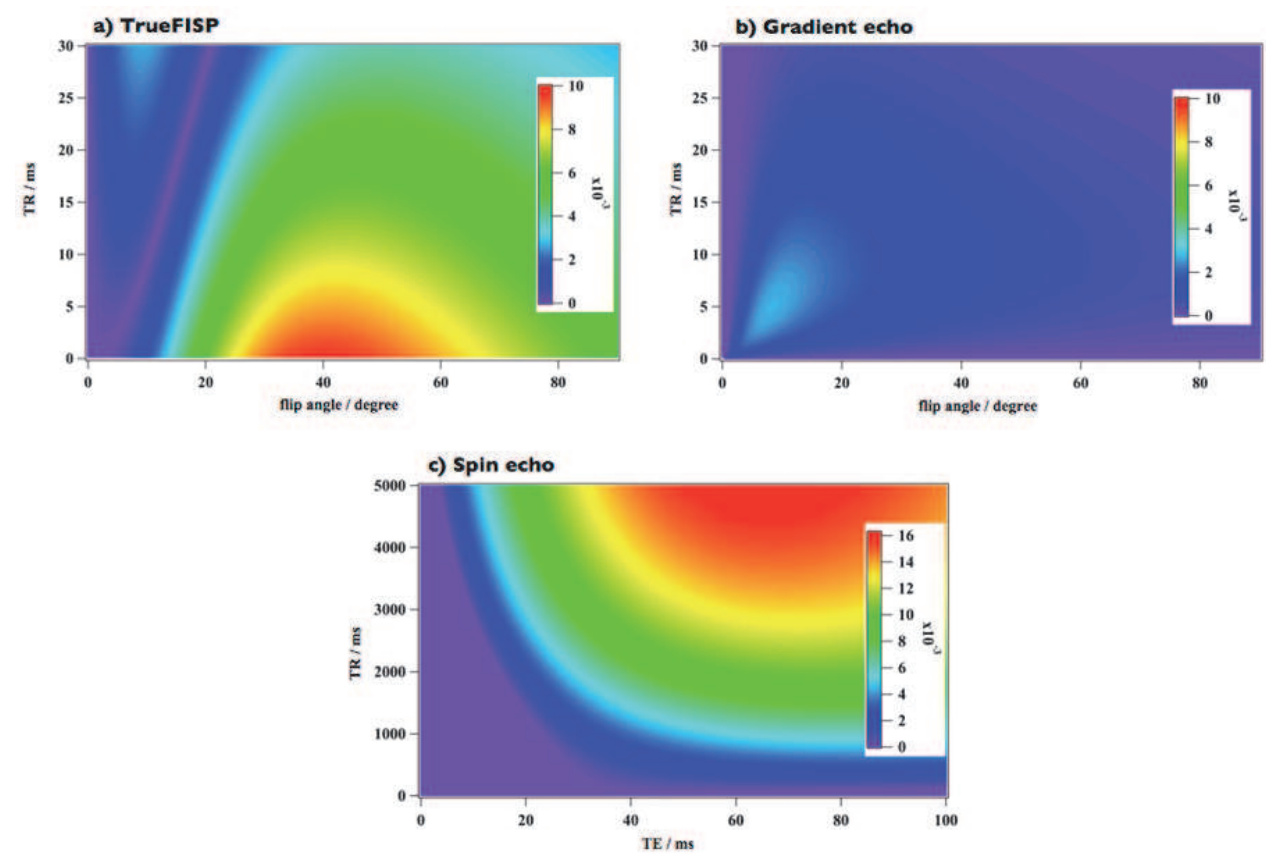

Fig. 6. Simulation of the contrast between tumor and brain using a) the 3D TrueFISP sequence, $b$ ) the $3 \mathrm{D}$ gradient-echo sequence, and c) the $2 \mathrm{D}$ spin-echo sequence. The contrast is estimated as a function of the flip angle and TR for the TrueFISP and gradient-echo sequences, and as a function of TE and TR for the spin-echo sequence.

\section{Results}

\subsection{Correction of TrueFISP banding artifacts}

TrueFISP images were acquired at $4.7 \mathrm{~T}$ using a cross-polarized antenna. A U87 glioma was implanted in a mouse, and imaging was performed 4 days later.

Fig. 7.a shows the image acquired using the TrueFISP sequence with a TE of $2.5 \mathrm{~ms}$, a flip angle of $35^{\circ}$, and a $\Delta \varphi$ value of $180^{\circ}$. As expected, black-signal bands are clearly visible in the image. These hinder visualization of parts of the animal's brain. Modifying the $\Delta \varphi$ value (to $0^{\circ}, 90^{\circ}$, and $270^{\circ}$ ) shifts the positions of the null-signal bands. For some of these values $\left(\Delta \varphi=270^{\circ}\right)$, the tumor is clearly visible, while for others $\left(\Delta \varphi=90^{\circ}\right)$, the null-signal zone covers the area containing the tumor, making all subsequent analyses of the tumor impossible. 


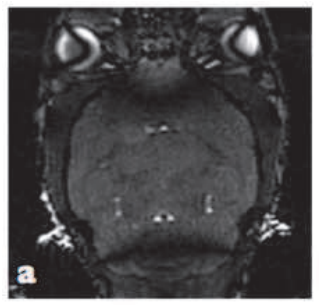

$\Delta \phi=180^{\circ}$

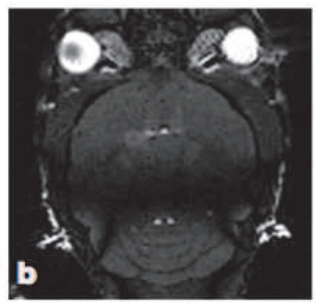

$\Delta \phi=0^{\circ}$

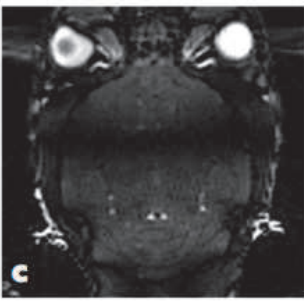

$\Delta \phi=90^{\circ}$

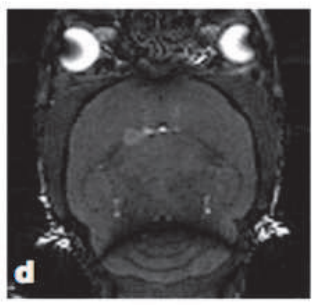

$\Delta \phi=270^{\circ}$

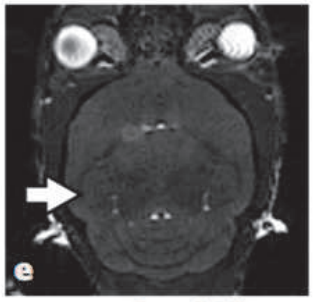

$e=\left(a^{2}+b^{2}\right)^{1 / 2}$

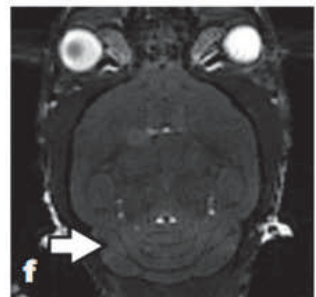

$f=\left(a^{2}+b^{2}+c^{2}\right)^{1 / 2}$

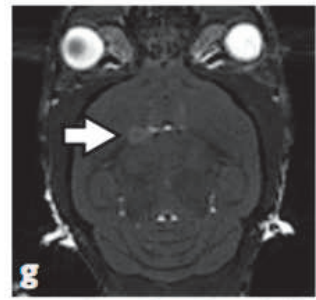

$g=\left(a^{2}+b^{2}+c^{2}+d^{2}\right)^{1 / 2}$

Fig. 7. Brain images from a mouse with an implanted tumor on day 4 after implantation. Images were obtained using the $3 \mathrm{D}$ TrueFISP sequence at $4.7 \mathrm{~T}$ and $\Delta \varphi=180^{\circ}(\mathrm{a}), 0^{\circ}(\mathrm{b}), 90^{\circ}$ (c), $270^{\circ}$ (d). Null-signal bands are clearly visible and shift in position. Image e was obtained by SOS reconstruction from $a$ ) and $b$ ). The arrow indicates a non-compensated low-signalintensity band. Image $f$ was obtained by reconstruction from $a), b$ ), and c). The arrow indicates a high-signal-intensity band. Finally, Image $g$ was obtained from the 4 images a), b), c), and d). The arrow shows the tumor, with a volume of less than $2 \mu \mathrm{L}$.

To eliminate these null-signal bands, the images acquired in a), b), c), and d) can be combined. To do so, various methods have been described in the literature, such as the sum of k-spaces, the sum of magnitude images, or the square root of the sum of squares of magnitude images. The latter method will be used here as it has been shown to be the most effective [14].

As shown in Images a-b, the black-signal bands do not appear to overlap. These two images were therefore combined to produce Image e. This eliminated the very pronounced nullsignal bands, and the tumor became clearly visible. Nevertheless, the signal was not homogeneous throughout the brain, and a band (indicated by the white arrow in Image e) shows a reduced signal intensity compared with other areas of the brain. This type of artifact appears in various positions for all types of combinations of 2 images (ac, ad, bc, etc.).

This low-signal-intensity band could be eliminated by adding a third image to the combination ( $a, b$, and $c)$. However, a band of slightly higher signal intensity (white arrow in Image f) appeared. The position of this band corresponds to the black-signal band in Image $\mathrm{d}$.

Finally, by adding Image $d$ to the final combination, Image $g$ was obtained $(g=(a 2+b 2+c 2$ $+\mathrm{d} 2) 1 / 2$. In this case, the brain appears perfectly homogeneous and the tumor is clearly visible. 


\subsection{Influence of flip angle on $\mathrm{S} / \mathrm{N}$ and contrast on TrueFISP imaging}

As shown in the simulations, for a TR of $5 \mathrm{~ms}$, the $\mathrm{S} / \mathrm{N}$ should be optimal with a flip angle between $15^{\circ}$ and $35^{\circ}$. On the other hand, contrast between tumor and brain should be optimal with a flip angle between $25^{\circ}$ and $55^{\circ}$. For a fixed TE, TrueFISP images were acquired with a flip angle between $8^{\circ}$ and $35^{\circ}$. Above this range, the $\mathrm{S} / \mathrm{N}$ is reduced and, more importantly, signals from sub-cutaneous fat and fluids (such as cerebrospinal fluid) become very intense. This leads to a strong reduction in signal dynamics for the brain and tumor, and generates images which are more difficult to interpret.

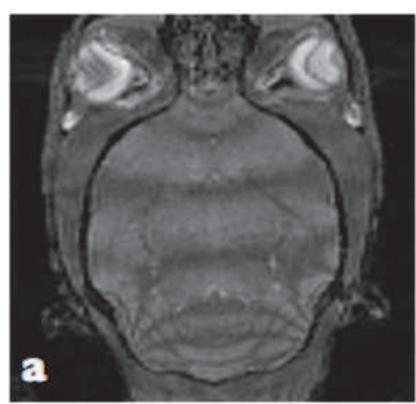

$\alpha=8^{\circ}$

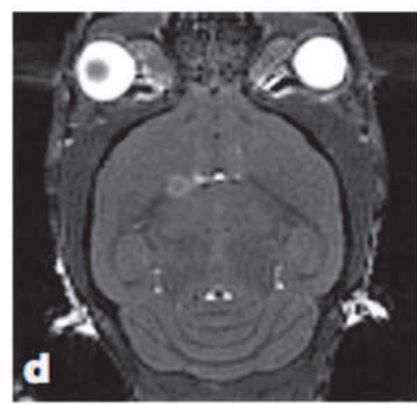

$\alpha=25^{\circ}$

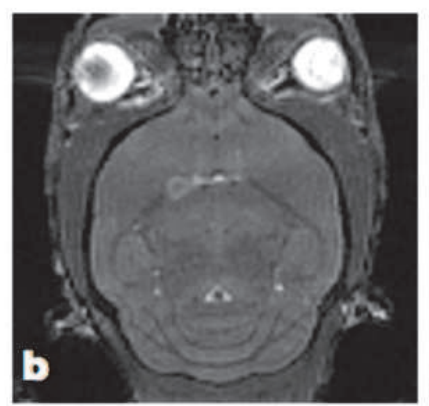

$\alpha=15^{\circ}$

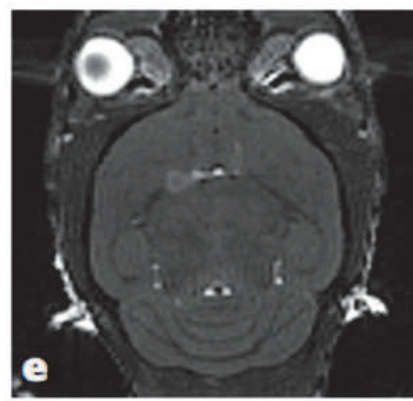

$\alpha=30^{\circ}$

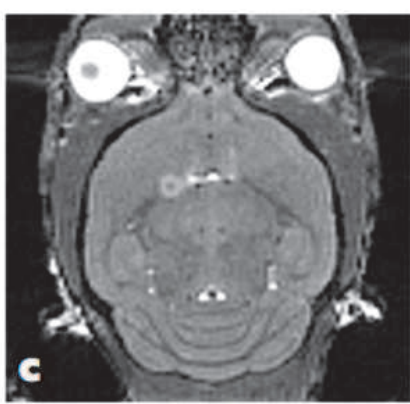

$\alpha=20^{\circ}$

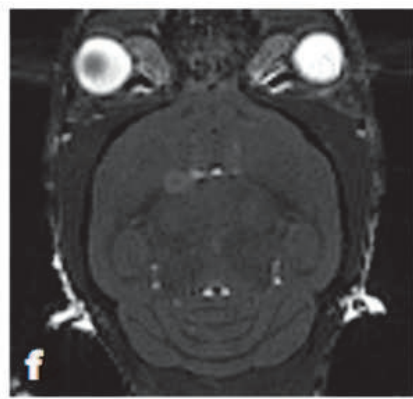

$\alpha=35^{\circ}$

Fig. 8. Images obtained using the 3D TrueFISP sequence after correction of banding artifacts according to the a flip angle. TE was set to $2.5 \mathrm{~ms}$.

The best contrast between brain and tumor was achieved at $35^{\circ}$. For $\alpha=25$ and $30^{\circ}$, good contrast was maintained, providing easy tumor detection, and the $\mathrm{S} / \mathrm{N}$ was increased. Below this, the signal for healthy brain was increased and the tumor became difficult to distinguish. An $8^{\circ}$ angle gave rise to more TrueFISP artifacts in different positions. At this value, reconstruction could not generate an image without null-signal bands, and the tumor became indistinguishable from the surrounding brain.

The table below gives the $\mathrm{S} / \mathrm{N}$ and contrast between the brain and tumor for the various flip angles. To summarize, flip angles between 25 and $35^{\circ}$ allow tumors to be segmented semiautomatically based on tissue contrast. 


\begin{tabular}{ccccc} 
TE = 2.5 ms & flip angle $\left(^{\circ}\right)$ & $\begin{array}{c}\text { S/N } \\
\text { Brain }\end{array}$ & $\begin{array}{c}\text { S/N } \\
\text { Tumor }\end{array}$ & Contrast \\
\cline { 2 - 5 } & 35 & 26.0 & 37.7 & 11.7 \\
30 & 29.8 & 40.3 & 10.5 \\
25 & 33.3 & 43.5 & 10.2 \\
20 & 46.3 & 56.6 & 10.3 \\
15 & 51 & 60.5 & 9.5 \\
8 & 57.6 & 57.8 & 0.2 \\
\hline
\end{tabular}

Table 1. S/N and contrast for brain and tumor, as a function of the flip angle, a. TE was set to $2.5 \mathrm{~ms}$.

\subsection{Influence of TE on S/N and contrast in TrueFISP imaging}

Using a low bandwidth generates images with a high S/N. However, a decrease in bandwidth causes an increase in the sequence's TE. The influence of this was measured over the range between 1.5 and $10 \mathrm{~ms}$ (with the flip angle set at $\left.35^{\circ}\right)$. With a low TE $(1.5 \mathrm{~ms})$, the contrast appeared better than in the reference image acquired at $2.5 \mathrm{~ms}$, but the $\mathrm{S} / \mathrm{N}$ was significantly reduced, and the signal from fatty tissue was increased due to its relatively low T1. This resulted in a lower-quality image. Interestingly, at higher TEs (5 and $10 \mathrm{~ms}$ ), the TrueFISP sequence generated few susceptibility artifacts compared with a FLASH sequence with the same TE (data not shown). However, at TE $=10 \mathrm{~ms}$, the tumor was no longer visible due to a strongly reduced contrast between brain and tumor.

As described in the table below, only a TE value of 2.5 ms provides a contrast greater than 10 , allowing the tumor to be segmented semi-automatically.

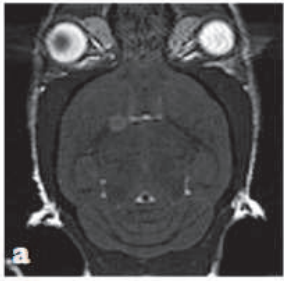

$\mathrm{TE}=1.5 \mathrm{~ms}$

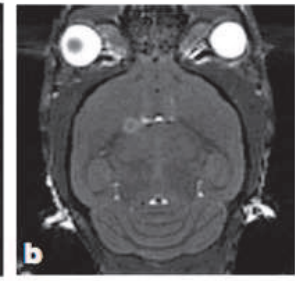

$\mathrm{TE}=\mathbf{2 . 5} \mathrm{ms}$

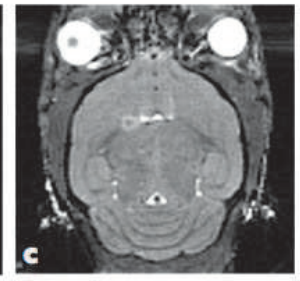

$\mathrm{TE}=\mathbf{5} \mathrm{ms}$

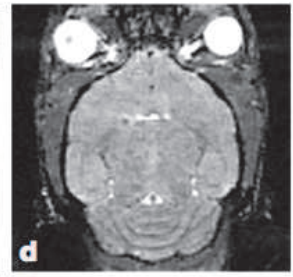

$\mathrm{TE}=10 \mathrm{~ms}$

Fig. 9. Images acquired using the 3D TrueFISP sequence after correction of banding artifacts based on TE. The flip angle was set to $35^{\circ}$.

\begin{tabular}{ccccc} 
flip angle $=35^{\circ}$ & TE & $\begin{array}{c}\mathrm{S} / \mathrm{N} \\
\text { Brain }\end{array}$ & $\begin{array}{c}\mathrm{S} / \mathrm{N} \\
\text { Tumor }\end{array}$ & Contrast \\
\hline 1.5 & 22.46 & 28.53 & 6 \\
2.5 & 26.0 & 37.7 & 11.7 \\
5 & 46.7 & 54.4 & 7.7 \\
10 & 38.6 & 41.9 & 3.3 \\
\hline
\end{tabular}

Table 2. S/N and contrast for brain and tumor, as a function of the echo time. The flip angle a was set at $35^{\circ}$. 


\subsection{Comparison between RARE and TrueFISP imaging}

The reference method for tumor detection by anatomic imaging is T2-weighted spin-echo imaging. Images were therefore acquired with a T2-weighted 2D RARE sequence. This sequence detects the tumor despite its small size (see Fig. 10d). In addition, as expected from simulations, contrast between the tumor and healthy brain is higher than with the TrueFISP sequence (Fig. 10a). The total acquisition time for the 2D RARE sequence was comparable with that for the 3D TrueFISP sequence. However, as the images were acquired in 2D by multi-slice imaging, reconstruction in other dimensions generates images with poor resolution and low quality (Figs. 10ef) compared with the 3D TrueFISP sequence (Figs. 10bc).

The two imaging methods were next used to measure tumor volume. With the TrueFISP sequence, the volume was estimated at $2.4 \mu \mathrm{L}$, while with the RARE sequence it was estimated at $4.3 \mu \mathrm{L}$. This overestimation of the volume with the RARE sequence is due to the low spatial resolution of this sequence in the third dimension. Actually, significant partialvolume effects are generated by this sequence and lead to volume-measurement errors.

A 3D RARE sequence validated the $2.4 \mu \mathrm{L}$ volume determined using the TrueFISP sequence. The 3D RARE image was acquired over 45 minutes.
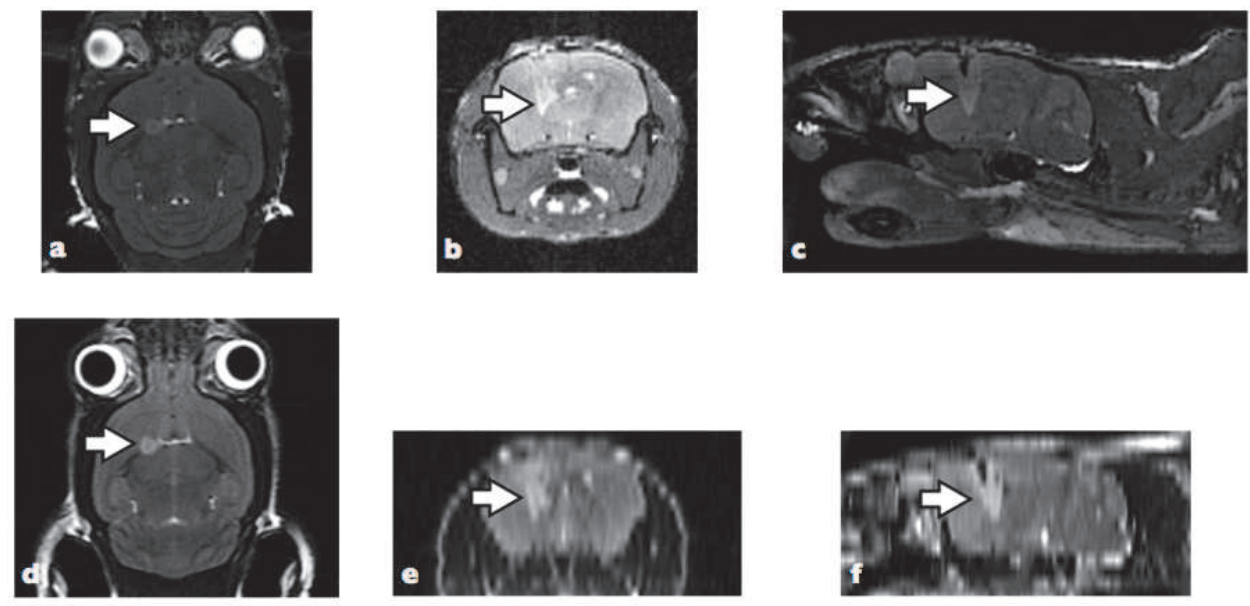

Fig. 10. 3D TrueFISP (a, b, and c) and multi-slice 2D RARE imaging (d, e, and $\mathrm{f}$ ) of a mouse with implanted glioma: a) \& d) sagittal sections; b) \& e) reconstruction of transverse sections; c) \& f) reconstruction of coronal sections. The white arrows indicate the tumor.

Volume overestimation based on 2D imaging was confirmed by using the two sequences to image a phantom consisting of a known volume of water. With the 2D RARE sequence, the error in volume measurement was around $20 \%$, while with the TrueFISP sequence the error was only $4 \%$. 


\subsection{Imaging at 9.4T}

Images were acquired with the same 2 sequences at $9.4 \mathrm{~T}$ on a mouse model of glioma. To suppress banding artifacts with the TrueFISP sequence, 8 images had to be acquired with 8 different $\Delta \varphi$ values $\left(180^{\circ}, 0^{\circ}, 90^{\circ}, 270^{\circ}, 45^{\circ}, 135^{\circ}, 225^{\circ}, 315^{\circ}\right)$.

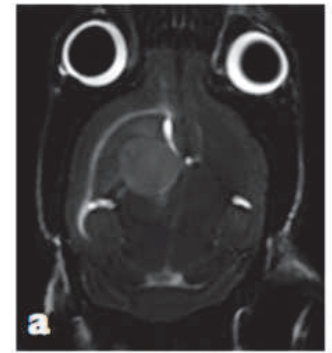

RARE

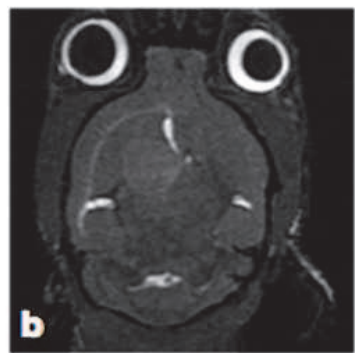

TrueFISP

Fig. 11. 2D RARE (a) and 3D TrueFISP (b) imaging at 9.4 T. With RARE imaging, the tumor is clearly visible, while TrueFISP provides a lower tumor/brain contrast.

With the RARE sequence, the tumor is clearly detectable and contrast is comparable with that of an image acquired at $4.7 \mathrm{~T}$ (Fig. 10a). For the TrueFISP sequence, the contrast is reduced, making it very difficult to distinguish the tumor from the remainder of the brain, except from the presence of structural heterogeneity. In this case, it is not possible to semiautomatically segment the tumor, and it is therefore impossible to measure its volume with good reproducibility. In addition, as demonstrated previously, small tumors may turn out to be undetectable.

\begin{tabular}{cccc} 
TE & $\begin{array}{c}\text { S/N } \\
\text { Brain }\end{array}$ & $\begin{array}{c}\text { S/N } \\
\text { Tumor }\end{array}$ & Contrast \\
\hline TrueFISP 4.7 T & 26.0 & 37.7 & 11.7 \\
RARE 4.7 T & 25.4 & 38.5 & 13.1 \\
TrueFISP 9.4 T & 24.6 & 28.3 & 3.7 \\
RARE 9.4 T & 18.9 & 32.2 & 13.3 \\
\hline
\end{tabular}

Table 3. S/N and contrast for brain and tumor, at $4.7 \mathrm{~T}$ and 9.4 T, with RARE and TrueFISP sequences.

\subsection{Longitudinal follow up of glioma volume at 4.7T}

The simulations and experiments performed above show that an implanted tumor can be unambiguously distinguished by 3D TrueFISP imaging when a TE of $2.5 \mathrm{~ms}$ is combined with a flip angle of $35^{\circ}$ for imaging at $4.7 \mathrm{~T}$. These parameters were therefore used for the longitudinal follow-up of tumor volume in an animal implanted with a U87-type glioma. Images were also acquired with the 2D RARE sequence to compare volume measurements. 
The tumor was easily detectable 7 days after implantation despite its small volume $(2 \mu \mathrm{L})$. The images obtained with the TrueFISP sequence are shown from day 9 after implantation. The animal was then followed up for approximately 30 days, with a 2 or 3-day interval between MRI scans. The increase in tumor diameter is visible in the images (Fig. 12). From $\mathrm{d} 25$, zones of black signal appear in the tumor's center, these are probably due to necrotic areas.

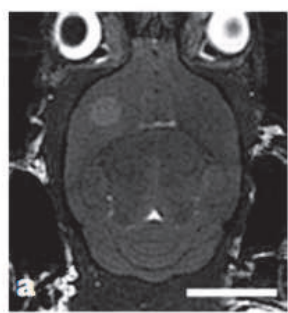

d9

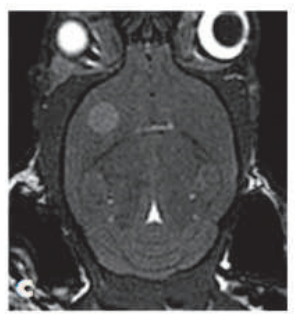

dII

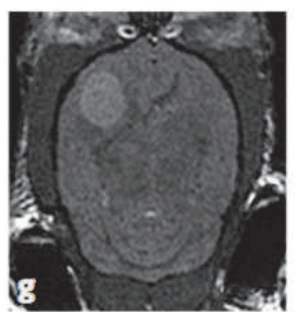

d2 I

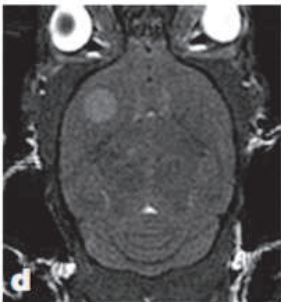

dI4

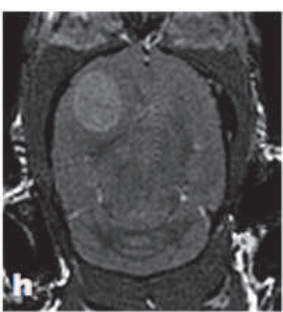

d23

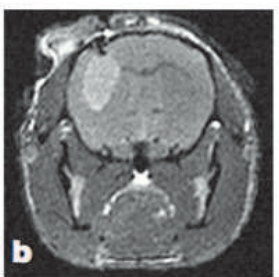

d9

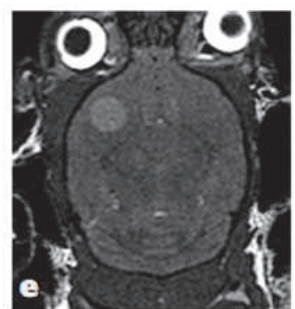

dI6

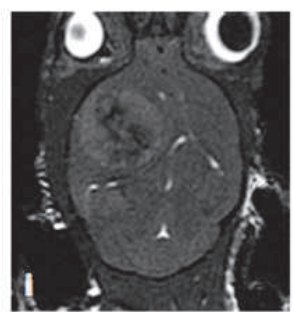

d25

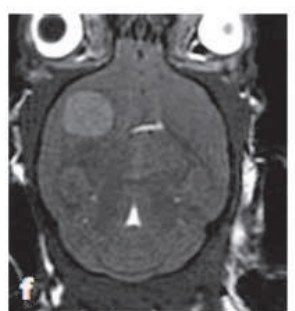

d18

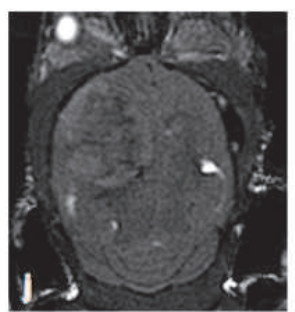

d28

Fig. 12. Longitudinal follow-up of tumor progression by 3D TrueFISP imaging at $4.7 \mathrm{~T}$. Images ab show the sagittal and transverse sections of the mouse brain at $\mathrm{d} 9$ after implantation. Images cj show tumor progression between $\mathrm{d} 11$ and $\mathrm{d} 28$.

Based on these 3D images (a-h), the tumor can readily be semi-automatically segmented, and the 3D volume could be reconstructed (Fig. 13). In Images i-j, the tumor/brain contrast is lower, but the tumor can still be manually delimited and its volume measured.

Based on these 3D reconstructions, tumor volume progression was reported as a function of time (Fig. 14). Tumor growth could thus be assessed, from its initial approximately $4 \mu \mathrm{L}$ volume up to its final $100 \mu \mathrm{L}$ volume, i.e. approximately one quarter of the animal's total brain volume. 
On the other hand, with the 2D RARE sequence, the tumor is also visible from day 7 after implantation. However, the volume measured based on images acquired with the RARE sequence is always greater than that measured by TrueFISP, and, importantly, very little difference is observed over the first 4 analysis times $(\mathrm{d} 9, \mathrm{~d} 11, \mathrm{~d} 14, \mathrm{~d} 16)$. This is probably due to a less precise measurement of the tumor volume, which, as indicated above, is due to very low spatial resolution in the third dimension.

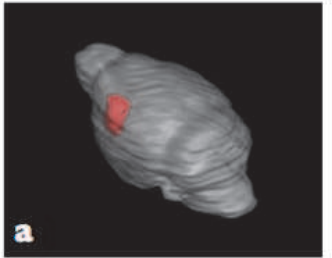

d9

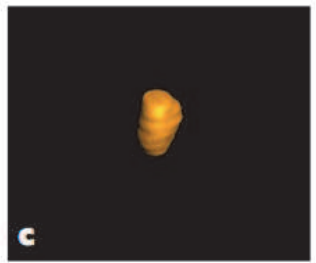

dII

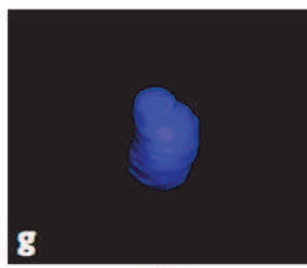

d2I

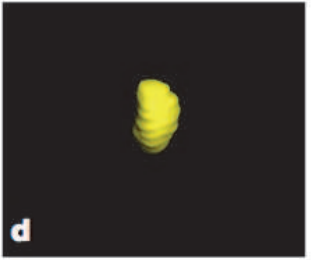

d14

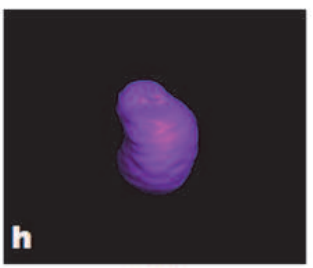

d23

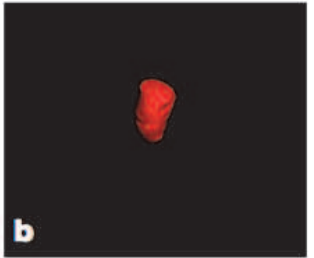

d9

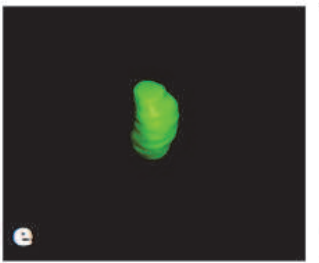

dI6

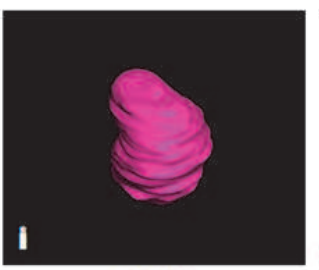

d25

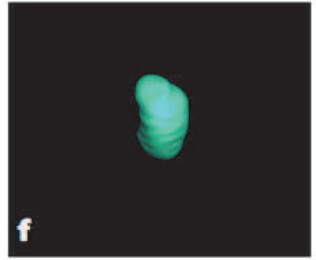

d18

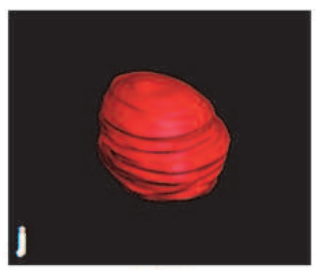

d28

Fig. 13. 3D reconstruction of the tumor by semi-automatic segmentation from the images shown in Figure 11. Image a: reconstruction of the whole brain (gray) and the tumor (red) at d9. Images bj: 3D representation of the tumor over time. The same arbitrary scale is used in all images. 


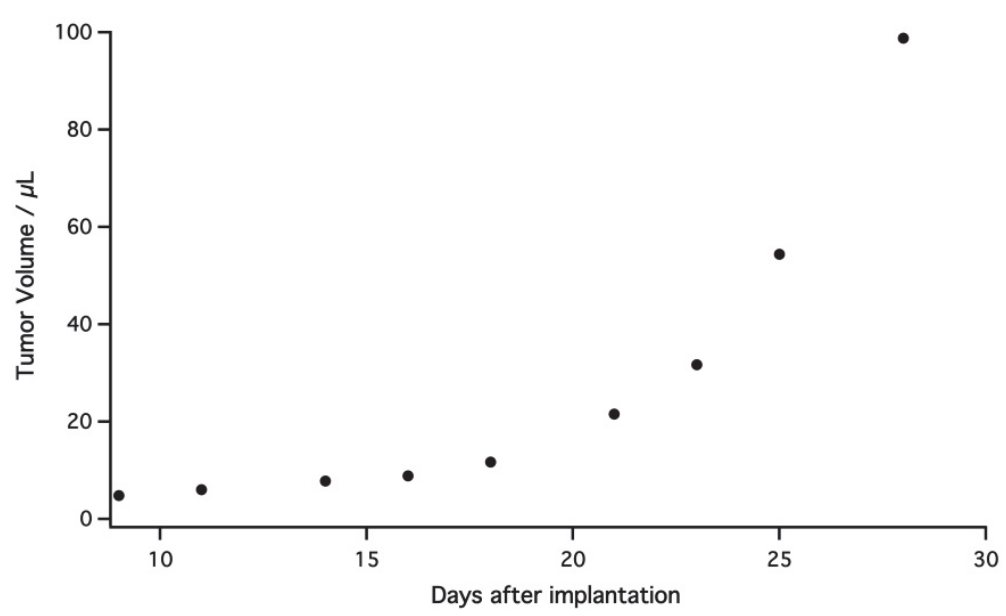

Fig. 14. Progression of tumor volume over time based on the volumes shown in Figure 13.

\section{Discussion and conclusion}

The aim of this chapter was to show how the 3D TrueFISP sequence can improve longitudinal follow-up of glioma volume in a given animal model.

This sequence is rarely used at high magnetic fields as it generates numerous artifacts known as banding artifacts [10-12]. However, the $\mathrm{S} / \mathrm{N}$ and contrast provided by this sequence can be useful in imaging small animals at high fields.

It has been shown that these artifacts can be easily corrected at $4.7 \mathrm{~T}$ and $9.4 \mathrm{~T}$ when studying mouse brains, and also for areas which are much more sensitive to movements and susceptibility artifacts, such as the heart $[8,13]$. To do so, the so-called 'alternating-phase RF pulse' method is used, in combination with reconstruction through calculation of the square root of the sum of squares of the magnitude signal [14]. As shown here, 4 phase steps are necessary to suppress artifacts at $4.7 \mathrm{~T}$, while at least 8 steps are required at $9.4 \mathrm{~T}$ [8]. The resulting image appears perfectly homogeneous in terms of signal, and no longer displays artifacts. The requirement for 4 images increases the total acquisition time, but this nevertheless remains much shorter than with a T2-weighted 3D RARE sequence (12 min vs. $45 \mathrm{~min})[8,15]$.

The 3D TrueFISP imaging sequence was compared, in terms of $\mathrm{S} / \mathrm{N}$ and contrast, with the more commonly used gradient-echo and spin-echo sequences. Simulations, which were confirmed by experimental results, indicate a clear superiority of this sequence in terms of $\mathrm{S} / \mathrm{N}$. As the sequence can be used with short TRs (like a gradient-echo sequence), it is perfectly adapted to 3D imaging, unlike spin-echo sequences which require long TRs. A high $\mathrm{S} / \mathrm{N}$ is obtained with the magnetization maintained in the transverse plane, thanks to a zero gradient sum at the end of each TR. This is much better than 'spoiling', which is used with the more common gradient-echo or FLASH sequences.

The TrueFISP sequence provides a much higher signal-to-noise ratio than a $2 \mathrm{D}$ accelerated spin-echo sequence. This is associated with better spatial resolution in the third dimension. Yet, the RARE sequence can also be used in 3D and also provides very high S/Ns. However, additional spatial encoding for the T2-weighted RARE spin echo has a drastic effect on the 
total acquisition time: good T2 weighting is required for tumor detection, and can only be obtained with TRs greater than 3,500 ms. This leads to a total acquisition time on the order of 1 hour, whereas around 10 minutes is adequate with the 2D sequence or the 3D TrueFISP sequence.

The TrueFISP sequence provides adequate contrast, allowing even very small tumors to be detected clearly, and semi-automatically segmented using generic image-analysis tools. However, as shown in both simulations and images, the contrast between the healthy brain and the tumor remains lower than that provided by the spin-echo sequence. This is due to TrueFISP-sequence weighting, which relies on the T1/T2 ratio, while the RARE sequence only uses T2 weighting. This has several consequences. In some cases, e.g. when the tumor becomes very large and heterogeneous, semi-automatic segmentation becomes difficult due to inadequate contrast with the healthy brain. Manual segmentation therefore becomes necessary, which may be both tedious and subjective. Nevertheless, the error rate when estimating even large volumes remains low.

Another consequence is that it is almost impossible to detect a glioma in the brain at fields higher than 4.7 $\mathrm{T}$ based on natural contrast. This is because the $\mathrm{T} 1$ and $\mathrm{T} 2$ values for the brain and tumor become almost identical, which limits the contrast at very high magnetic fields. The T2-weighted RARE sequence still works perfectly at these higher fields.

Thus, for this type of application, the TrueFISP sequence at $9.4 \mathrm{~T}$ or greater provides little advantage over other, more commonly used sequences. However, at low fields, the S/N and contrast provided by the TrueFISP sequence make it particularly interesting. It has already been shown with specific instruments that it allows tumors to be easily detected in mice based on natural contrast, and that it provides an excellent signal-to-noise ratio at clinical magnetic fields (1.5 $\mathrm{T}$ and $3 \mathrm{~T})[16,17]$.

In terms of spatial resolution, the advantages of 3D compared with 2D imaging are obvious. Three-dimensional imaging results in much smaller voxel sizes with comparable acquisition times, while maintaining a high $\mathrm{S} / \mathrm{N}$. This requires the addition of a phase-encoding table in the slice direction, i.e. a much greater number of lines read in the Fourier volume. The advantages of sequences with short TRs thus become obvious. As shown, the TrueFISP sequence also provides a much greater precision when measuring tumor volume, in particular for small tumors. With the 2D RARE sequence, it is impossible to precisely measure volume changes in the first days following tumor implantation. With the 3D TrueFISP sequence, this information is readily available.

In addition, as the sequence is relatively rapid in terms of total acquisition time, it can be repeated at very short intervals to follow tumor progression, even in relatively fragile mice. The total examination, between preparation of the animal and imaging, lasts less than 20 minutes. This makes it possible to study animals on a daily basis. This is much more difficult when contrast agents must be injected, or with acquisition times of around 1 hour, as with 3D RARE sequences [15].

To conclude, the 3D TrueFISP sequence can be easily used to follow tumor progression in a small animal model imaged at $4.7 \mathrm{~T}$. Thanks to a particularly high $\mathrm{S} / \mathrm{N}$, artifact-free images can be acquired, with excellent spatial resolution and good tumor/healthy brain contrast. The total acquisition time remains under 15 minutes, thus offering precise longitudinal follow-up of tumor volume. Thus, this sequence could be used to noninvasively validate the efficacy of new genetic or pharmacological treatments for glioma.

At lower magnetic fields, the sequence has also demonstrated its efficacy. In contrast, it is currently not applicable at $9.4 \mathrm{~T}$ or higher to precisely measure tumor volumes. 


\section{References}

[1] Bock NA, Zadeh G, Davidson LM, et al. High-resolutionlongitudinal screening with magnetic resonance imaging in a murine brain cancer model. Neoplasia 2003;5:546554.

[2] Moats RA, Velan-Mullan S, Jacobs R, et al. Micro-MRI at 11.7T of a murine brain tumor model using delayed contrast enhancement. Mol Imaging 2003;2:150-158 [erratum: Mol Imaging 2003;2(4)].

[3] Miraux S, Lemiere S, Pineau R, et al. Inhibition of FGF receptor activity in glioma implanted into the mouse brain using the tetra- cyclin-regulated expression system. Angiogenesis 2004;7:105-113.

[4] Sun Y, Mulkern RV, Schmidt K, et al. Quantification of water dif- fusion and relaxation times of human U87 tumors in a mouse model. NMR Biomed 2004;17:399-404.

[5] Quesson B, Bouzier AK, Thiaudiere E, Delalande C, Merle M, Ca- nioni P. Magnetization transfer fast imaging of implanted glioma in the rat brain at 4.7T: interpretation using a binary spin-bath model. J Magn Reson Imaging 1997;7:1076-1083.

[6] Hennig J, Nauerth A, Friedburg H. RARE imaging: a fast imaging method for clinical MR. Magn Reson Med 1986;3:823-833.

[7] Oppelt A, Graumann R, Barfu H, Fischer H, Hartl W, Schajor W. FISP - a new fast MRI sequence. Electromedica 1986;54:15-18.

[8] Miraux S, Massot P, Ribot EJ, Franconi JM, Thiaudiere E. 3D TrueFISP imaging of mouse brain at 4.7T and 9.4T. J Magn Reson Imaging 2008;28:497-503.

[9] De Coene B, Hajnal JV, Gatehouse P, Longmore DB, White SJ, Oatridge A, Pennock JM, Young IR, Bydder GM. MR of the brain using fluid-attenuated inversion recovery (FLAIR) pulse sequences. AJNR Am J Neuroradiol. 1992 NovDec;13(6):1555-64.

[10] Haacke EM, Wielopolski PA, Tkach JA, Modic MT. Steady-state free precession imaging in the presence of motion: application for im- proved visualization of the cerebrospinal fluid. Radiology 1990;175: 545-552.

[11] Zur Y, Wood ML, Neuringer LJ. Motion-insensitive, steady-state free precession imaging. Magn Reson Med 1990;16:444 - 459.

[12] Vasanawala SS, Pauly JM, Nishimura DG. Linear combination steady-state free precession MRI. Magn Reson Med 2000;43:82-90. 11. Casselman JW, Kuhweide R, Deimling M, Ampe W, Dehaene I, Meeus L. Constructive interference in steady state-3DFT MR imaging of the inner-ear and cerebellopontine angle. AJNR Am J Neuroradiol 1993;14:47-57.

[13] Miraux S, Calmettes G, Massot P, Lefrancois W, Parzy E, Muller B, Arsac LM, Deschodt-Arsac V, Franconi JM, Diolez P, Thiaudie`re E. 4D retrospective black blood trueFISP imaging of mouse heart. Magn Reson Med 2009; 62: 1099-1105.

[14] Vasanawala SS, Pauly JM, Nishimura DG. Linear combination steady-state free precession MRI. Magn Reson Med 2000; 43: 82-90.

[15] Natt O, Watanabe T, Boretius S, Radulovic J, Frahm J, Michaelis T. High-resolution 3D MRI of mouse brain reveals small cerebral structures in vivo. J Neurosci Methods 2002;120:203-209. 
[16] Bernas LM, Foster PJ, Rutt BK. Imaging iron-loaded mouse glioma tumors with bSSFP at 3 T. Bernas LM, Foster PJ, Rutt BK. Magn Reson Med. 2010 Jul;64(1):23-31.

[17] Ribot EJ, Martinez-Santiesteban FM, Simedrea C, Steeg PS, Chambers AF, Rutt BK, Foster PJ, In Vivo Single Scan Detection of Both Iron-Labeled Cells and Breast Cancer Metastases in the Mouse Brain Using Balanced Steady-State Free Precession Imaging at 1.5 T. J Magn Reson Imaging 2011 Jul;34(1):231-8. 


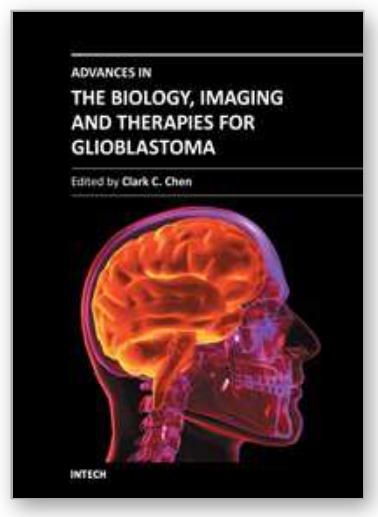

\author{
Advances in the Biology, Imaging and Therapies for Glioblastoma \\ Edited by Prof. Clark Chen
}

ISBN 978-953-307-284-5

Hard cover, 424 pages

Publisher InTech

Published online 09, November, 2011

Published in print edition November, 2011

This book is intended for physicians and scientists with interest in glioblastoma biology, imaging and therapy. Select topics in DNA repair are presented here to demonstrate novel paradigms as they relate to therapeutic strategies. The book should serve as a supplementary text in courses and seminars as well as a general reference.

\title{
How to reference
}

In order to correctly reference this scholarly work, feel free to copy and paste the following:

Emeline Julie Ribot, Line Pourtau, Philippe Massot, Pierre Voisin, Eric Thiaudiere, Jean- Michel Franconi and Sylvain Miraux (2011). 3D TrueFISP MRI Provides Accurate Longitudinal Measurements of Glioma Volumes in Mice, Advances in the Biology, Imaging and Therapies for Glioblastoma, Prof. Clark Chen (Ed.), ISBN: 978953-307-284-5, InTech, Available from: http://www.intechopen.com/books/advances-in-the-biology-imagingand-therapies-for-glioblastoma/3d-truefisp-mri-provides-accurate-longitudinal-measurements-of-gliomavolumes-in-mice

\section{INTECH}

open science | open minds

\section{InTech Europe}

University Campus STeP Ri

Slavka Krautzeka 83/A

51000 Rijeka, Croatia

Phone: +385 (51) 770447

Fax: +385 (51) 686166

www.intechopen.com

\section{InTech China}

Unit 405, Office Block, Hotel Equatorial Shanghai

No.65, Yan An Road (West), Shanghai, 200040, China

中国上海市延安西路65号上海国际贵都大饭店办公楼 405 单元

Phone: +86-21-62489820

Fax: +86-21-62489821 
(C) 2011 The Author(s). Licensee IntechOpen. This is an open access article distributed under the terms of the Creative Commons Attribution 3.0 License, which permits unrestricted use, distribution, and reproduction in any medium, provided the original work is properly cited. 